225

\section{Subarachnoid Hemorrhage (SAH) with ST-Segment Elevation}

Morita $H$, Kuroiwa T, Tanabe $H$

Osaka Mishima Critical Care Medical Center

Osaka, Japan

Objective: There are few reports of subarachnoid hemorrhage (SAH) accompanied by ST-segment elevation. Coronary angiography (CAG) and left ventriculography (LVG) in five patients with SAH and ST-segment elevation are reported.

Methods: The subjects consisted of five patients, four men, and one woman with mean age of 52 years, who suffered from $\mathrm{SAH}$ with ST-segment elevation on admission. The CAG and LVG were performed immediately after the head CT and cerebral angiography and measurement of serum CK-MB.

Results: All patients had a ruptured cerebral aneurysm. The CT findings revealed all the cases to be of Fisher Class 4 . The LVG showed apical wall motion decreased in spite of preservation of the basal wall motion. No significant stenoses or spasm of the coronary arteries were observed by CAG. The CK-MB titre was not significantly elevated.

Conclusion: As no abnormal findings were present in the $\mathrm{CAG}$ or serum CK-MB, the cause of differential movement between basal and apical portion of the left ventricle is considered to be a reflection of different quantity of sympathetic nerve distribution within the $\mathrm{LV}$ wall.

\section{7}

\section{The Relationship between Impact Speed and Injury Severity Score (ISS) in Motor Vehicle-Pedestrian Accidents}

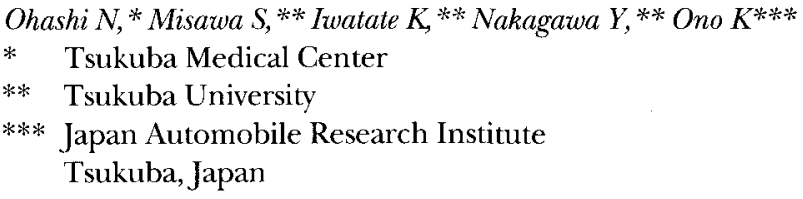

Materials: A total of 103 pedestrian victims involved in 98 traffic accidents were analyzed. All of these patients were hit by the front-end of small-sized sedans while they were crossing the street.

\section{Results: [Figure]}

\section{ISS}

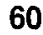

\section{0}

$$
\begin{aligned}
& 40+++++ \\
& 200+t_{00}+t_{00} \\
& 10 \circ 0.000 \\
& \begin{array}{ccc}
10 & 0 & 0 \\
0000 & 0000000
\end{array} \\
& \begin{array}{lllllll}
10 & 20 & 30 & 40 & 50 & 60 & 70
\end{array} \\
& \text { Impact Speed km/h }
\end{aligned}
$$

Conclusion: Injury severity scores (ISS) correlate with impact speed. The mortality rate is markedly high at an impact speed above 45 kilometers/hour.

\section{8}

\section{Handy Container of Surgical Equipment Serving as Formaldehyde Gas-Sterilizing Box}

Shirakawa $Y$, Ogli $K$, Nogaya I

Kagawa Medical School

Kagawa, Japan

Objective: To design a compact container system which is convenient for the storage, transport, and sterilization of a small set of surgical equipment in the field during disasters. Such an device must meet special requirements: 1) always available on standby; 2) short sterilization cycle for repeated use; 3) ease of handling and maintenance by non-professional personnel; 4) low-cost to run; and 5) operate without energy sources.

A formaldehyde gas-sterilization substance (EFGEN) was selected as a sterilizing agent, which, if stored in an air-tight container, keeps its ability to elaborate formaldehyde gas absorbed by plaster granules for more than six months. The prototype that was developed consists of two stainless steel boxes. The small sterilizer box $(150 \times 150 \times 30 \mathrm{~mm})$ is fixed to the lid of the main one $(300 \times 40 \times 80 \mathrm{~mm})$. Both chambers are connected through tiny windows, which can be closed by a sliding shutter. Effects of sterilization were studied by measuring the biological indicators.

Results: One and one-half hours might be enough for complete sterilization.

\section{Table-Results of Cultures}

Duration of

Sterilization

$<45$ min

$60 \mathrm{~min}$

$>75 \mathrm{~min}$

B. stearothermophilus
positive
negative
negative

B. subtilis
positive
positive
negative

Conclusion: Although further studies are required on the safety and utility of the system, this handy tool potentially could be applied in many situations. 